\title{
ARMAZENAMENTO DE PÊSSEgOS (Prunus persica (L.) Batsch), cv. CHIRIPÁ, EM ATMOSFERA CONTROLADA ${ }^{1}$
}

\author{
GILMAR ANTÔNIO NAVA² \& AURI BRACKMANN ${ }^{3}$
}

\begin{abstract}
RESUMO-O experimento foi conduzido com o objetivo de avaliar diferentes pressões parciais de $\mathrm{O}_{2} \mathrm{e} \mathrm{CO}_{2}$ durante o armazenamento em atmosfera controlada (AC) sobre a qualidade físico-química de pêssegos, cv. Chiripá. As pressões parciais de gases avaliadas foram: $0,8 \mathrm{kPaO}_{2} / 3 \mathrm{kPaCO}_{2} ; 1 \mathrm{kPaO}_{2} / 3 \mathrm{kPaCO}_{2} ; 1 \mathrm{kPaO}_{2} / 4 \mathrm{kPaCO}_{2} ; 1 \mathrm{kPaO}_{2} / 5 \mathrm{kPaCO}_{2} ; 2 \mathrm{kPaO} / 6 \mathrm{kPaCO}_{2} ; 2 \mathrm{kPaO}_{2} / 7 \mathrm{kPaCO}_{2}, 2 \mathrm{kPaO}_{2} / 8 \mathrm{kPaCO}_{2}$, ar $/$ $10 \mathrm{kPaCO}_{2}$ e tratamento-controle, mantido sob armazenamento refrigerado (AR). O delineamento experimental utilizado foi o inteiramente casualizado, com quatro repetições de 20 frutos. As avaliações foram realizadas após 4 e 8 semanas de armazenamento a $-0,5^{\circ} \mathrm{C} \pm$ $0,2^{\circ} \mathrm{C}$, mais 2 dias a $20^{\circ} \mathrm{C}$. Os resultados evidenciaram que o controle de atmosfera reduz a degradação da clorofila da epiderme e a perda de peso, porém tem pouco efeito sobre a firmeza, teor de sólidos solúveis totais (SST) e acidez titulável dos frutos. A pressão parcial de $1 \mathrm{kPa}$ de $\mathrm{O}_{2}$, associada a $3 \mathrm{kPa}$ de $\mathrm{CO}_{2}$, reduz a manifestação dos sintomas de lanosidade durante 8 semanas de armazenamento. A incidência de podridões dos frutos é reduzida quando armazenados sob a condição de $2 \mathrm{kPa}$ de $\mathrm{O}_{2}$, associada a $8 \mathrm{kPa}$ de $\mathrm{CO}_{2}$. Tanto o armazenamento refrigerado quanto o armazenamento sob atmosfera controlada mantêm boa qualidade dos frutos por quatro semanas a $-0,5^{\circ} \mathrm{C}$, mais 2 dias a $20^{\circ} \mathrm{C}$. Oito semanas de armazenamento em AC é um período muito prolongado para uma satisfatória manutenção da qualidade de pêssegos da cultivar Chiripá. A elevada incidência de podridões e de lanosidade são as causas que mais contribuem para a redução da qualidade de pêssegos, cv. Chiripá, durante períodos prolongados de armazenamento.
\end{abstract}

Termos de indexação: Qualidade, oxigênio, gás carbônico, lanosidade.

\section{THE STORAGING OF PEACHES cv. CHIRIPÁ, IN CONTROLLED ATMOSPHERE}

\begin{abstract}
The experiment was carried out with the objective to evaluate different partial pressures of $\mathrm{O}_{2}$ and $\mathrm{CO}_{2}$ in controlled atmosphere storage on the quality 'Chiripá' peaches. The evaluated controlled atmospheres conditions were: $0,8 \mathrm{kPaO}_{2} / 3 \mathrm{kPaCO}_{2}$; $1 \mathrm{kPaO}_{2} / 3 \mathrm{kPaCO}_{2} ; 1 \mathrm{kPaO}_{2} / 4 \mathrm{kPaCO}_{2} ; 1 \mathrm{kPaO}_{2} / 5 \mathrm{kPaCO}_{2} ; 2 \mathrm{kPaO}_{2} / 6 \mathrm{kPaCO}_{2} ; 2 \mathrm{kPaO}_{2} / 7 \mathrm{kPaCO}_{2}, 2 \mathrm{kPaO} / 8 \mathrm{kPaCO}_{2}$, air/10kPaCO and control treatment in cold storage. The experiment design was entirely randomized with 4 replications of 20 fruits. The evaluations were accomplished after 4 and 8 weeks of storage at $-0,5^{\circ} \mathrm{C} \pm 0,2^{\circ} \mathrm{C}$ plus 2 days at $20^{\circ} \mathrm{C}$. The results showed that controlled atmosphere reduces the degradation of the chlorophyll and the weight loss, but has a little effect on firmness, soluble solids and acidity. During long term storage $1 \mathrm{kPa}$ of $\mathrm{O}_{2}$, associated with $3 \mathrm{kPa}$ of $\mathrm{CO}_{2}$, prevents occurence of wooliness. The fruits deterioration was reduced in $2 \mathrm{kPa}$ of $\mathrm{O}_{2}$, associated with $8 \mathrm{kPa}$ of $\mathrm{CO}_{2}$. The cold storage, as well as controlled atmosphere storage, maintains good fruit quality for 4 weeks at $-0,5^{\circ} \mathrm{C}$ plus 2 days shelf life. Eight weeks controlled atmosphere storage period is too long to maintain good quality of peaches. The high incidence of rot and wooliness are the main causes that reduce peaches quality, Chiripá cv., during long term storage.
\end{abstract}

Index terms: Quality, oxygen, carbonic gas, woolliness.

\section{INTRODUÇÃO}

A crescente expansão do cultivo de pêssegos para consumo "in natura" vem aumentando significativamente a oferta do produto. $\mathrm{O}$ 'Chiripá' é uma cultivar de maturação tardia que a torna bastante apta para o armazenamento, visando ao abastecimento do mercado por um período maior. No entanto, o pêssego é extremamente perecível e não se presta a períodos prolongados de armazenamento a frio. De acordo com Haerter \& Araujo (1994), esta alta perecibilidade está associada às altas taxas respiratórias, ocasionando curta vida de armazenamento e maior suscetibilidade à perda de qualidade e valor alimentar do fruto. Segundo Holland et al. (1994), o pêssego pode ser armazenado em temperaturas de $-1^{\circ} \mathrm{C}$ a $2^{\circ} \mathrm{C}$, dependendo da cultivar; entretanto, nestas temperaturas, algumas cultivares desenvolvem sintomas de danos pelo frio, os quais limitam o tempo de vida póscolheita dos frutos. Para Luchsinger \& Walsh (1998), a lanosidade é o principal dano pelo frio de importância comercial em pêssegos e nectarinas.

A redução dos níveis de $\mathrm{O}_{2}$ e/ou elevação dos níveis de $\mathrm{CO}_{2}$ retarda o amadurecimento dos frutos (Lana \& Finger, 2000), diminui o metabolismo de pigmentos, de compostos fenólicos e voláteis (Beaudry, 1999), reduz a síntese e a ação do etileno sobre o metabolismo dos frutos e a ocorrência de podridões (Brackmann \& Chitarra, 1998). Segundo Lana \& Finger (2000), os níveis mínimos de $\mathrm{O}_{2}$ e máximos de $\mathrm{CO}_{2}$ são limitados pela sensi-

1 (Trabalho 118/2001). Recebido: 15/06/2001. Aceito para publicação: 20/12/2001. Trabalho apresentado como requisito parcial para obtenção do título de Mestre em Agronomia na Universidade Federal de Santa Maria-UFSM;

2 Engo Agr $^{-}$Msc. em Agronomia, Professor Substituto da Escola Agrotécnica Federal de Rio do Sul-Unidade de Ensino Descentralizada de Dois Vizinhos/PR.

3 Eng $\underline{0}$ Agr ${ }^{\circ}$ Dr. em Fisiologia Pós-Colheita, Professor Adjunto do Departamento de Fitotecnia da UFSM. Autor para correspondência: Campus Universitário, Santa Maria/RS, CEP: 97105-900.

Rev. Bras. Frutic., Jaboticabal - SP, v. 24, n. 2, p. 328-332, agosto 2002 
bilidade dos tecidos à indução de respiração anaeróbica e injúria por $\mathrm{CO}_{2}$, respectivamente. Entretanto, de acordo com Ke et al. (1993), pequeno estresse por baixo $\mathrm{O}_{2}$ e/ou elevado $\mathrm{CO}_{2}$ não induz metabolismo fermentativo. Nestas condições, a taxa de respiração e o consumo de reservas são reduzidos, mantendo uma melhor qualidade dos produtos, além de prolongar o período de armazenamento.

A firmeza de polpa e a coloração da epiderme, de acordo com Rushing \& Dinamarca (1993), são as duas características de qualidade em pêssegos mais consistentemente afetadas pelo controle de atmosfera. Segundo os mesmos autores, o armazenamento em atmosfera controlada também controla podridões em frutos de caroço durante o amadurecimento a $20^{\circ} \mathrm{C} \mathrm{e}$, de maneira geral, reduz o surgimento de lanosidade, principalmente quando o período de armazenamento é prolongado.

Segundo Eris et al. (1994), pêssegos, cv. Hale Haven, podem ser mantidos sob armazenamento em AC na condição de $2 \mathrm{kPa}$ de $\mathrm{O}_{2}$, associada a 5 e $10 \mathrm{kPa}$ de $\mathrm{CO}_{2}$, por um período de 30 dias, a $0^{\circ} \mathrm{C}$ e $90-95 \%$ de umidade relativa (UR), mais 4 dias a 20 $23^{\circ} \mathrm{C}$. Por outro lado, Chitarra \& Chitarra (1990) afirmam que pêssegos podem ser armazenados até 6 semanas em atmosfera controlada com $1-2 \mathrm{kPa}$ de $\mathrm{O}_{2}$, mais $3-5 \mathrm{kPa}$ de $\mathrm{CO}_{2}$, na temperatura de -1 a $0,5^{\circ} \mathrm{C}$.

As pesquisas demonstram que as cultivares de pêssegos respondem de forma diferenciada às pressões parciais de gases, bem como ao período de armazenamento. O presente trabalho objetivou avaliar diferentes pressões parciais de $\mathrm{O}_{2} \mathrm{e} \mathrm{CO}_{2}$ sobre a qualidade físico-química de pêssegos, cv. Chiripá.

\section{MATERIAL E MÉTODOS}

O experimento foi realizado no Núcleo de Pesquisa em Pós-colheita (NPP) do Departamento de Fitotecnia da Universidade Federal de Santa Maria. O material experimental constou de frutos da cultivar Chiripá, oriundos de um pomar comercial localizado em Farroupilha-RS, sendo que as amostras experimentais foram homogeneizadas e armazenadas em minicâmaras experimentais de ferro galvanizado hermeticamente vedadas, com volume de 70 e $185 \mathrm{~L}$.

As condições de atmosfera controlada foram estabelecidas um dia após a instalação do experimento, mediante diluição do $\mathrm{O}_{2}$ através da injeção de nitrogênio no interior das minicâmaras, o qual foi obtido por um gerador do tipo PSA ("Pressure Swing Adsorption"). As pressões parciais de $\mathrm{CO}_{2}$ foram estabelecidas por meio da injeção do gás proveniente de cilindros sob alta pressão para o interior das minicâmaras. $\mathrm{O}$ monitoramento das concentrações de gases foi realizado diariamente com o auxílio de analisadores de gases de fluxo contínuo. As pressões parciais de $\mathrm{O}_{2}$ e $\mathrm{CO}_{2}$ testadas foram: $0,8 \mathrm{kPaO}_{2} /$ $3 \mathrm{kPaCO}_{2} ; 1 \mathrm{kPaO}_{2} / 3 \mathrm{kPaCO}_{2} ; 1 \mathrm{kPaO}_{2} / 4 \mathrm{kPaCO}_{2} ; 1 \mathrm{kPaO}_{2} / 5 \mathrm{kPaCO}_{2}$; $2 \mathrm{kPaO}_{2} / 6 \mathrm{kPaCO}_{2} ; 2 \mathrm{kPaO}_{2} / 7 \mathrm{kPaCO}_{2}, 2 \mathrm{kPaO}_{2} / 8 \mathrm{kPaCO}_{2}, \mathrm{Ar} /$ $10 \mathrm{kPaCO}_{2}$ e tratamento-controle mantido sob armazenamento refrigerado (AR), em que $1 \mathrm{kPa}$ é equivalente a $1 \%$ ao nível do mar. A umidade relativa das minicâmaras foi mantida em torno de $95 \mathrm{e}$ 97\% para o armazenamento refrigerado e para os tratamentos com controle de atmosfera, respectivamente.

Em cada minicâmara, foram colocadas oito amostras de 20 frutos para as avaliações, que foram realizadas com quatro repetições para cada avaliação, após 4 e 8 semanas de armazenamento, a $-0,5^{\circ} \mathrm{C} \pm 0,2^{\circ} \mathrm{C}$, mais dois dias a $20^{\circ} \mathrm{C}$, a fim de simular o período de beneficiamento e comercialização dos frutos. Os parâmetros avaliados foram: firmeza de polpa, determinada pela resistência da polpa a um penetrômetro com ponteira de $5 / 16$ polegadas $(7,9 \mathrm{~mm})$; teor de sólidos solúveis totais (SST), obtido por refratometria a partir do suco extraído de cada amostra de 20 frutos; acidez titulável, obtida através da titulação de $10 \mathrm{~mL}$ de suco, obtido das amostras de frutos, diluído em $100 \mathrm{~mL}$ de água destilada e titulada com uma solução de $\mathrm{NaOH}$ a $0,1 \mathrm{~N}$ até $\mathrm{pH} 8,1$; coloração da epiderme, determinada com o auxílio de um colorímetro pelo sistema CIE L*a*b*, que representa uma escala de cores, em que "L" indica a intensidade de claridade (preto ao branco), "a" indica a progressão da cor verde à vermelha e " $b$ " indica a progressão da cor azul à amarela; incidência de podridões, determinada pela contagem dos frutos que apresentaram lesões de tamanho superior a $5 \mathrm{~mm}$ de diâmetro e com características típicas de ataque de fungos; perda de peso, obtida pela diferença de peso das amostras no momento da instalação do experimento e o peso final das mesmas na saída das câmaras; incidência de lanosidade, determinada de forma subjetiva através do pressionamento dos frutos entre os dedos e pela visualização direta da presença ou ausência de suco e/ou polpa farinácea. A intensidade do dano foi expressa por meio de um índice de lanosidade, onde se consideraram: índice $\mathbf{1}=$ frutos com ausência de suco e polpa farinácea (totalmente lanosos); índice $\mathbf{2}=$ frutos com teor médio de suco (pouco a medianamente lanosos); índice 3= frutos com muito suco (não-lanosos); produção de etileno, obtida por cromatografia gasosa por meio de cromatógrafo equipado com coluna paropak e detector de ionização de chama, na temperatura de $90 ; 120$ e $200^{\circ} \mathrm{C}$ para o injetor, coluna e detector, respectivamente; respiração, determinada pela produção diária de $\mathrm{CO}_{2}$ através de um analisador de fluxo contínuo de gases.

Os dados foram analisados segundo um delineamento inteiramente casualizado, com 4 repetições. Havendo diferença significativa entre os tratamentos pelo teste $\mathrm{F}$, as médias foram comparadas pelo teste de Duncan, em nível de 5\% de probabilidade de erro.

\section{RESULTADOS E DISCUSSÃO}

A firmeza da polpa foi bruscamente reduzida em relação à firmeza na colheita em todos as condições de armazenamento testadas (Tabela 1); entretanto, entre a $4^{\mathrm{a}}$ e a $8^{\mathrm{a}}$ semana de armazenamento (Tabela 2), essa redução não foi observada. Com exceção dos tratamentos com $2 \mathrm{kPa}$ de $\mathrm{O}_{2}$, associado a 7 e $8 \mathrm{kPa}$ de $\mathrm{CO}_{2}$, que se manteve inalterada, verificou-se aumento na firmeza de polpa dos frutos. Este fenômeno, segundo Purvis (1993) e Luchsinger (2000), é devido à geleificação das pectinas parcialmente degradadas da parede celular dos frutos. No entanto, o efeito das pressões parciais de $\mathrm{O}_{2}$ e $\mathrm{CO}_{2}$ sobre a firmeza dos frutos foi mais evidente após 8 semanas de armazenamento (Tabela 2), quando a firmeza de polpa se manteve mais elevada na condição de $0,8 \mathrm{kPa}$ de $\mathrm{O}_{2}$, associada a $3 \mathrm{kPa}$ de $\mathrm{CO}_{2}$, seguida pelos tratamentos com $1 \mathrm{kPa}$ de $\mathrm{O}_{2}$, associados a 3 e $4 \mathrm{kPa}$ de $\mathrm{CO}_{2}$. Após 4 semanas de armazenamento, os frutos mantidos sob armazenamento refrigerado, enriquecido com $10 \mathrm{kPa}$ de $\mathrm{CO}_{2}$, apre- 
sentaram um índice de lanosidade superior aos demais tratamentos testados (Tabela 1). Verificou-se, após este período de armazenamento, que o controle da concentração de gases teve pequeno efeito na redução dos sintomas de lanosidade, quando somente os tratamentos com $1 \mathrm{kPa}$ de $\mathrm{O}_{2}$, associado a $4 \mathrm{kPa}$ de $\mathrm{CO}_{2}$ e $2 \mathrm{kPa}$ de $\mathrm{O}_{2}$, associado a $8 \mathrm{kPa}$ de $\mathrm{CO}_{2}$ promoveram uma pequena inibição dos sintomas, proporcionando um teor mais elevado de suco nos frutos. Segundo Zoffoli et al. (1997), a lanosidade em pêssegos, cv. 'Elegant Lady', foi reduzida unicamente quando mantidos na pressão parcial de $1 \mathrm{kPa}$ de $\mathrm{O}_{2}$, associada a $30 \mathrm{kPa}$ de $\mathrm{CO}_{2}$; entretanto, nesta condição, ocorreu falta de sabor e aroma ("off flavor") devido ao acúmulo de etanol. Ao final de 8 semanas de armazenamento, a condição de $1 \mathrm{kPa}$ de $\mathrm{O}_{2}$; associada a $3 \mathrm{kPa}$ de $\mathrm{CO}_{2}$, contraditoriamente aos resultados obtidos após 4 semanas de armazenamento, reduziu os sintomas de lanosidade, onde os frutos apresentaram alto teor de suco (Tabela 2). Este resultado parece indicar que, nesta condição de armazenamento, houve um restabelecimento do amadurecimento normal dos frutos em função do aumento da atividade das enzimas responsáveis pela degradação das pectinas da parede celular dos tecidos, o que está em acordo com Purvis (1993). Este autor afirma que o componente crítico do armazenamento em AC, para reduzir a severidade dos danos pelo frio, parece ser o
$\mathrm{CO}_{2}$ nas pressões parciais inferiores a $5 \mathrm{kPa}$, por manter a capacidade dos frutos em produzir níveis adequados de enzimas pectolíticas e manter a integridade das membranas celulares nas temperaturas de amadurecimento. Para Von Mollendorff et al. (1992), o incremento no conteúdo de suco nos estádios mais avançados de maturação é, provavelmente, devido ao prolongamento da atividade das Poligalacturonases (PG) sobre as pectinas de alto peso molecular, transformando-as em pectinas de baixo peso molecular, as quais não formam gel.

Observou-se, nos frutos lanosos, após 4 semanas de armazenamento, que a lanosidade é um processo reversível. A reversão dos sintomas de lanosidade ocorreu, parcialmente, após 2-3 dias na temperatura de amadurecimento de $20^{\circ} \mathrm{C}$, onde os frutos apresentaram menor firmeza e um restabelecimento parcial do teor de suco (dados não apresentados). Estes resultados concordam com Zhou et al. (2000), Luchsinger (2000) e Von Mollendorff et al. (1992), que afirmam também que a temperatura e a firmeza de polpa durante o amadurecimento são fatores envolvidos no processo de reversão.

Verificou-se considerável aumento na incidência de podridões ao longo do período de armazenamento, principalmente nos tratamentos submetidos às menores pressões parciais de $\mathrm{O}_{2}$ e $\mathrm{CO}_{2}$ testadas (Tabelas 3 e 4). O tratamento com $2 \mathrm{kPa}$

TABELA 1 - Qualidade físico-química de pêssegos, cv. Chiripá, armazenados durante 4 semanas em atmosfera controlada a $-0,5^{\circ} \mathrm{C}$, mais 2 dias a $20^{\circ}$ C. Santa Maria-RS, 2000.

\begin{tabular}{|c|c|c|c|c|}
\hline $\begin{array}{c}\mathrm{O}_{2} / \mathrm{CO}_{2} \\
(\mathrm{kPa})\end{array}$ & $\begin{array}{l}\text { Firmeza } \\
\text { (Newton) }\end{array}$ & $\begin{array}{c}\text { Lanosidade } * * \\
(1-3)\end{array}$ & $\begin{array}{c}\text { Acidez Titul. } \\
(\text { cmol/L })\end{array}$ & $\begin{array}{c}\text { Sólidos solúveis } \\
\left({ }^{\circ} \mathrm{Brix}\right)\end{array}$ \\
\hline Colheita & 69,4 & - & 8,09 & 12,7 \\
\hline AR & $23,3 \quad a^{*}$ & 2,35 & 4,53 & 13,1 ab \\
\hline $\mathrm{Ar} / 10$ & 23,5 a & 1,80 & $5,05 \quad \mathrm{ab}$ & 13,3 a \\
\hline $1,0 / 3,0$ & $19,4 \mathrm{ab}$ & 2,09 & $5,02 \mathrm{abc}$ & $13,0 \mathrm{ab}$ \\
\hline $1,0 / 4,0$ & $16,2 \quad b$ & 2,66 a & 4,96 abc & $12,7 \quad b$ \\
\hline $1,0 / 5,0$ & 23,3 a & $2,34 \quad b$ & 5,27 a & $13,1 \mathrm{ab}$ \\
\hline $2,0 / 6,0$ & 24,5 a & $2,34 \quad b$ & 4,74 & $12,9 \mathrm{ab}$ \\
\hline $2,0 / 7,0$ & $21,8 \mathrm{ab}$ & 2,40 & 4,93 & $12,9 \mathrm{ab}$ \\
\hline $2,0 / 8,0$ & 24,9 a & $2,53 \mathrm{ab}$ & 4,73 & $13,1 \quad a b$ \\
\hline
\end{tabular}

*Tratamentos com médias não seguidas pela mesma letra na vertical diferem, pelo teste de Duncan, em nível de 5\% de probabilidade de erro; AR= Armazenamento Refrigerado.

**Índice de lanosidade: $1=$ frutos com ausência de suco e polpa farinhenta (totalmente lanosos); $2=$ frutos com pouco suco (pouco a medianamente lanosos);

$3=$ frutos altamente suculentos (não-lanosos).

TABELA 2 -Qualidade físico-química de pêssegos, cv Chiripá, armazenados durante 8 semanas em atmosfera controlada a - $0,5^{\circ} \mathrm{C}$ mais 2 dias a $20^{\circ}$ C. Santa Maria-RS, 2000.

\begin{tabular}{|c|c|c|c|c|}
\hline $\begin{array}{c}\mathrm{O}_{2} / \mathrm{CO}_{2} \\
(\mathrm{kPa})\end{array}$ & $\begin{array}{l}\text { Firmeza } \\
\text { (Newton) }\end{array}$ & $\begin{array}{c}\text { Lanosidade } * * \\
(1-3)\end{array}$ & $\begin{array}{l}\text { Acidez Titul. } \\
(\mathrm{cmol} / \mathrm{L})\end{array}$ & $\begin{array}{c}\text { Sólidos solúveis } \\
\left({ }^{\circ} \mathrm{Brix}\right)\end{array}$ \\
\hline $0,8 / 3,0$ & 43,5 a * & $2,43 \quad b$ & $4,53 \mathrm{Ab}$ & $12,5 \mathrm{a}$ \\
\hline $1,0 / 3,0$ & 35,1 & 2,85 a & $4,33 \mathrm{Ab}$ & 12,6 a \\
\hline $1,0 / 4,0$ & 33,9 & $2,31 \quad b c$ & $4,49 \mathrm{Ab}$ & $12,5 \mathrm{a}$ \\
\hline $1,0 / 5,0$ & 27,3 & 1,99 & $4,59 \mathrm{Ab}$ & $12,7 \mathrm{a}$ \\
\hline $2,0 / 6,0$ & 28,9 & 1,90 & 4,75 a & 12,6 a \\
\hline $2,0 / 7,0$ & 18,6 & 2,20 & $4,08 \quad B$ & $12,6 \mathrm{a}$ \\
\hline $2,0 / 8,0$ & 20,7 & 1,85 & $4,13 \quad \mathrm{~B}$ & $12,7 \mathrm{a}$ \\
\hline
\end{tabular}

*Tratamentos com médias não seguidas pela mesma letra na vertical diferem, pelo teste de Duncan, em nível de $5 \%$ de probabilidade de erro.

** Índice de lanosidade: $1=$ frutos com ausência de suco e polpa farinhenta (totalmente lanosos); $2=$ frutos com pouco suco (pouco a medianamente lanosos); $3=$ frutos altamente suculentos (não-lanosos). 
TABELA 3 - Qualidade físico-química de pêssegos, cv. Chiripá, armazenados durante 4 semanas em atmosfera controlada, a - $0,5^{\circ} \mathrm{C}$ mais 2 dias a $20^{\circ}$ C. Santa Maria-RS, 2000.

\begin{tabular}{|c|c|c|c|c|c|}
\hline $\begin{array}{c}\mathrm{O}_{2} / \mathrm{CO}_{2} \\
(\mathrm{k} \mathrm{Pa})\end{array}$ & $\begin{array}{c}\operatorname{Cor} \\
\left(\mathbf{a}^{*}+\mathbf{b}^{*}\right)\end{array}$ & Podri & ã o & $\begin{array}{r}\text { Perda } I \\
(\%)\end{array}$ & es o \\
\hline Colbeita & 25,4 & - & & - & \\
\hline A R & 34,0 a $*$ & 17,8 & b c & 1,42 & $\mathrm{a}$ \\
\hline $\mathrm{Ar} / 10$ & $31,1 \quad b$ & 16,5 & $\mathrm{bc}$ & 0,96 & $\mathrm{bc}$ \\
\hline $1,0 / 3,0$ & 31,7 & 29,3 & $a b$ & 1,13 & b \\
\hline $1,0 / 4,0$ & 32,2 & 34,7 & $\mathrm{a}$ & 0,65 & $\mathrm{~d}$ \\
\hline $1,0 / 5,0$ & 31,5 & 26,8 & $a b c$ & 0,76 & $\mathrm{~cd}$ \\
\hline $2,0 / 6,0$ & 31,9 & 16,6 & b c & 0,85 & $\mathrm{~cd}$ \\
\hline $2,0 / 7,0$ & 31,8 & 15,1 & $\mathrm{bc}$ & 0,79 & $\mathrm{~cd}$ \\
\hline $2,0 / 8,0$ & 31,3 & 13,9 & $\mathrm{c}$ & 0,95 & $\mathrm{bc}$ \\
\hline
\end{tabular}

*Tratamentos com médias não seguidas pela mesma letra na vertical diferem, pelo teste de Duncan, em nível de 5\% de probabilidade de erro; $\mathrm{AR}=$ Armazenamento Refrigerado; ${ }^{1} \mathrm{CIE} \mathrm{L} * \mathrm{a} * \mathrm{~b} *$

TABELA 4 - Qualidade físico-química de pêssegos, cv Chiripá, armazenados durante 8 semanas em atmosfera controlada, a - $0,5^{\circ} \mathrm{C}$ mais 2 dias a $20^{\circ}$ C. Santa Maria-RS, 2000.

\begin{tabular}{|c|c|c|c|}
\hline $\begin{array}{c}\mathrm{O}_{2} / \mathrm{CO}_{2} \\
(\mathrm{kPa})\end{array}$ & $\begin{array}{c}\operatorname{Cor}^{1} \\
\left(\mathbf{a}^{*}+\mathbf{b}^{*}\right)\end{array}$ & $\begin{array}{c}\text { Podridão } \\
(\%)\end{array}$ & $\begin{array}{c}\text { Perda Peso } \\
(\%)\end{array}$ \\
\hline $0,8 / 3,0$ & $32,3 \quad \mathrm{c} *$ & $67,5 \quad a$ & $0,68 \quad \mathrm{bc}$ \\
\hline $1,0 / 3,0$ & $34,0 \quad a b$ & 72,5 a & 1,07 abc \\
\hline $1,0 / 4,0$ & $32,2 \quad \mathrm{c}$ & $55,7 \mathrm{ab}$ & 0,67 \\
\hline $1,0 / 5,0$ & 31,5 & 44,5 & 0,64 \\
\hline $2,0 / 6,0$ & $33,7 \mathrm{ab}$ & 35,7 & $1,12 \mathrm{ab}$ \\
\hline $2,0 / 7,0$ & 34,2 a & 35,2 & 1,04 abc \\
\hline $2,0 / 8,0$ & $32,8 \quad b c$ & 23,2 & $1,27 \mathrm{a}$ \\
\hline
\end{tabular}

*Tratamentos com médias não seguidas pela mesma letra na vertical diferem, pelo teste de Duncan, em nível de $5 \%$ de probabilidade de erro; ${ }^{1}$ CIE $\mathrm{L}^{*} \mathrm{a}{ }^{*} \mathrm{~b}{ }^{*}$.

de $\mathrm{O}_{2}$, associado a $8 \mathrm{kPa}$ de $\mathrm{CO}_{2}$, mostrou ser a melhor condição de armazenamento para a prevenção da incidência de podridões (Tabela 3); entretanto, não diferindo dos tratamentos com $1 \mathrm{kPa}$ de $\mathrm{O}_{2}$, associado a $5 \mathrm{kPa}$ de $\mathrm{CO}_{2}$ e $2 \mathrm{kPa}$ de $\mathrm{O}_{2}$, associado a 6 e $7 \mathrm{kPa}$ de $\mathrm{CO}_{2}, \mathrm{Ar}+10 \mathrm{kPaCO}$, e do tratamento-controle. Estes resultados parecem estar relacionados ao efeito do alto nível de $\mathrm{CO}_{2}$ sobre o crescimento de microorganismos causadores de podridões, principalmente fungos, pois, nestas condições de armazenamento, não se dispõe de evidências concretas da influência direta do nível de $\mathrm{O}_{2}$ sobre o desenvolvimento de vários fungos causadores de podridões em pêssegos (Agar et al., 1990; Brackmann et al., 1996). Resultados semelhantes foram obtidos após oito semanas de armazenamento com os tratamentos com $2 \mathrm{kPa}$ de $\mathrm{O}_{2}$, associados a 6, 7 e $8 \mathrm{kPa}$ de $\mathrm{CO}_{2}$ (Tabela 4). A alta incidência de podridões nos tratamentos com atmosfera controlada parece estar relacionada à elevada umidade relativa no interior da minicâmara, a qual foi mantida em torno de $97 \%$.

A perda de peso, de modo geral, foi menor nos tratamentos em AC (Tabela 3), a qual pode ser explicada pela menor atividade respiratória e, principalmente, pelo efeito da menor transpiração dos frutos em conseqüência da UR mais elevada nas minicâmaras de $\mathrm{AC}$ em relação à câmara de $\mathrm{AR}$. Entre os tratamentos com controle de AC, praticamente não se observaram diferenças significativas na perda de peso dos frutos (Tabelas 3 e 4 ).

A acidez titulável foi maior, após 4 semanas de armazenamento, nas pressões parciais de $1 \mathrm{kPa}$ de $\mathrm{O}_{2}$, associadas a $3 ; 4$ e $5 \mathrm{kPa}$ de $\mathrm{CO}_{2}$ e no tratamento em $\mathrm{AR}$ enriquecido com $10 \mathrm{kPa}$ de $\mathrm{CO}_{2}$ (Tabela 1). Estes resultados podem ser explicados, em parte, pela menor taxa respiratória dos frutos durante o armazenamento (dados não mostrados) e, conseqüentemente, menor utilização dos ácidos orgânicos como fonte de energia para o processo respiratório. Após 8 semanas de armazenamento, praticamente não se observaram diferenças significativas entre os tratamentos (Tabela 4 ).

De maneira geral, houve um pequeno aumento no teor de SST nas 4 primeiras semanas de armazenamento em relação ao teor no momento da colheita, com pequena redução posterior até o final do período de armazenamento (Tabelas 1 e 2). Porém, os tratamentos testados tiveram pequeno efeito sobre o teor de sólidos solúveis totais dos frutos ao longo do período de armazenamento.

A cor de fundo verde da epiderme dos frutos foi significativamente degradada durante as 4 primeiras semanas de armazenamento em relação à cor de fundo na colheita, sofrendo pequena alteração a partir deste período até o final do armazenamento (Tabelas 3 e 4). Contudo, a cor de fundo da epiderme manteve-se mais verde em todos os tratamentos com controle de atmosfera após 4 semanas de armazenamento (Tabela 3). Isto, possivelmente, ocorreu pela redução da atividade enzimática, principalmente das clorofilases sob o processo de degradação da clorofila e pela menor síntese de outros pigmentos. Estes resultados concordam com os deWankier et al. (1970). Após 8 semanas de armazenamento, a cor de fundo da epiderme manteve-se mais verde nos tratamentos com pressões parciais de $0,8 \mathrm{kPa}$ de $\mathrm{O}_{2}$, associada a $3 \mathrm{kPa}$ de $\mathrm{CO}_{2}$ e $1 \mathrm{kPa}$ de $\mathrm{O}_{2}$, associada a 4 e $5 \mathrm{kPa}$ de $\mathrm{CO}_{2}$ (Tabela 4), mostrando a eficiência do baixo nível de $\mathrm{O}_{2}$ e alto nível de $\mathrm{CO}_{2}$ na inibição da degradação das clorofilas (Brackmann, 1984; Chitarra \& Chitarra, 1990; Awad, 1993). 


\section{CONCLUSÕES}

1. O controle de atmosfera reduz a degradação de clorofila da epiderme, bem como a perda de peso, porém tem pouco efeito sobre a firmeza, teor de SST e acidez titulável dos frutos.

2. A pressão parcial de $1 \mathrm{kPa}$ de $\mathrm{O}_{2}$, associada a $3 \mathrm{kPa}$ de $\mathrm{CO}_{2}$, previne a manifestação dos sintomas de lanosidade, quando o período de armazenamento é superior a 4 semanas.

A pressão parcial de $2 \mathrm{kPa}$ de $\mathrm{O}_{2}$ e $8 \mathrm{kPa}$ de $\mathrm{CO}_{2}$ reduz a incidência de podridões até 8 semanas de armazenamento.

3. Tanto o armazenamento refrigerado quanto o armazenamento sob atmosfera controlada mantêm uma boa qualidade dos frutos por 4 semanas a $-0,5^{\circ} \mathrm{C}$, mais 2 dias a $20^{\circ} \mathrm{C}$. $\mathrm{O}$ armazenamento por 8 semanas em AC é um período muito prolongado para uma satisfatória manutenção da qualidade de pêssegos da cultivar Chiripá.

4. A elevada incidência de podridões e de lanosidade é o fator que mais contribui para a redução da qualidade de pêssegos, cv. Chiripá, durante 4 semanas de armazenamento. Após 8 semanas de armazenamento, estes problemas agravaram-se, deixando os frutos sem condições de comercialização.

\section{REFERÊNCIAS BIBLIOGRÁFICAS}

AGAR, T.; GARCIA, J.M.; MIEDTKE, U.; STREIF, J. Effect of high $\mathrm{CO}_{2}$ and low $\mathrm{O}_{2}$ concentrations on the growth of Botrytis cinerea at different temperatures. Gartenbauwissenschaft, Berlin, v.55, n.5, p.219-222, 1990.

AWAD, M. Fisiologia Pós-Colheita de Frutos. São Paulo: Nobel, 1993, 114p.

BEAUDRY, R.M. Effect of $\mathrm{O}_{2}$ and $\mathrm{CO}_{2}$ partial pressure on selected phenomena affecting fruit and vegetable quality. Postharvest Biology and Technology, Amsterdam, v.15, p.293303, 1999.

BRACKMANN, A. Frigoconservação contínua e intermitente de pêssegos (Prunus persica (L.) Batsch), cultivares Convênio e Capdeboscq, em atmosfera convencional e modificada. 1984. 96f. Dissertação (Mestrado) - Universidade Federal de Pelotas, Pelotas, 1984.

BRACKMANN, A.; CHITARRA, A.B. Atmosfera controlada e atmosfera modificada. In: Armazenamento e processamento de produtos agrícolas. Lavras: UFLA/SBEA, 1998. p.133-170.

BRACKMANN, A.; SAQUET, A. A.; VEIGA, V.V.; BORTOLUZ, L. Efeito das concentrações de $\mathrm{CO}_{2}$ e $\mathrm{O}_{2}$ no crescimento e esporulação de Penicillium expansum (Link.) Thom, in vitro. Revista Brasileira de Agrociência, Pelotas, v.2, n.3, p.147-150, 1996.

CHITARRA, M.I.F.; CHITARRA, A.B. Pós-colheita de frutos e hortaliças: fisiologia e manuseio. Lavras: ESAL/FAEPE, 1990. 320 p.

ERIS, A.; TÜRKBEN, C.; ÖZER, M.H. A research on controlled atmosphere (CA) storage of peach cv. Hale Haven. Acta
Horticulturae, Wageningen, n.368, p.767-776, 1994.

HAERTER, J.A.; ARAÚJO, P.J. Influência do ponto de colheita e da temperatura de armazenamento na evolução do etileno em pêssegos. In: CONGRESSO BRASILEIRO DE FRUTICULTURA, 13., 1994, Salvador. Anais... v.3, p.895-896.

HOLLAND, N.; CHITARRA, M.I.F.; CHIITARRA, A.B. Conservação pós- colheita de pêssegos: Interação entre cálcio e temperatura. In: CONGRESSO BRASILEIRO DE FRUTICULTURA, 13., 1994, Salvador. Anais... v.3, p.897-898.

KE, D.Y.; MATEOS, M.; KADER, A.A. Regulation of fermentative metabolism in fruits and vegetables by controlled atmospheres. In: INTERNATIONAL CONTROLLED ATMOSPHERE RESEARCH CONFERENCE, 6., 1993, Ithaca. Proceedings... v.1, p.63-77.

LANA, M.M.; FINGER, F.L. Atmosfera Modificada e Controlada. Aplicação na Conservação de Produtos Hortícolas. Brasília: Embrapa Hortaliças, 2000. 34p.

LUCHSINGER, L. Avanços na conservação de frutas de caroço. In: SIMPÓSIO INTERNACIONAL DE FRUTAS DE CAROÇO PÊSSEGOS, NECTARINAS E AMEIXAS, 1., 2000, Porto Alegre. Anais... p. 95-104.

LUCHSINGER, L.; WALSH, C.S. Chilling injury of peach fruitt during storage. Acta Horticulturae, Wageningen, n.464, p. 473477, 1998 .

PURVIS, A.C. Effects of short- term CA storage on cell wall polysaccharides during subsequent ripening of peaches. In: INTERNATIONAL CONTOLLED ATMOSPHERERESEARCH CONFERENCE, 6., 1993, Ithaca. Proceedings... v.1, p. 418-424.

RUSHING, J.W.; DINAMARCA, A. Simulated shipment of peaches, plums, and nectarines under controlled atmospheres. In: INTERNATIONAL CONTROLLED ATMOSPHERE RESEARCH CONFERENCE, 6., 1993, New York. Proceedings... v.1, p.202-222.

Von MOLLENDORFF, L.J.; JACOBS, G.; VILLIERS. O.T. Cold storage influences internal characteristics of nectarines during ripening. HortScience, Alexandria, v.27, n.12, p.1295-1297, 1992.

WANKIER, B.N.; SALUNKHE, D.K.; CAMPBELL, W.F. Effects of controlled atmosphere storage on biochemical changes in apricot and peach fruit. Journal of the American Society of Hortultural Science., Alexandria, v.95, n.5, p.604-609, 1970.

ZOFFOLI, J. P.; RODRIGUEZ, J., ALDUNCE, P., CRISOSTO, C. $H$. Development of high concentration carbon dioxide modified atmosphere packging systems to maintain peach quality. In: INTERNATIONAL CONTROLLED ATMOSPHERE RESEARCH CONFERENCE, 7., 1997, California. Proceedings... v.3, p.37-45.

ZHOU, H.W.; DONG, L.; BEN-ARIE, R.; LURIE, S. The role of ethylene in preventing wooliness in nectarines. In: INTERNATIONALCONFERENCEONPOSTHARVEST SCIENCE, 4., 2000, Jerusalem. Abstracts... Tel Aviv, 2000. p. 59. 